\title{
Utilisation du développement embryolarvaire de l'huitre creuse crassostrea gigas comme outil de diagnostic de la toxicité de substances pures et de mélanges complexes \\ Use of embryo-larval development stage of the oyster Crassostrea gigas as a toxicity assessment tool for pure substances and complex mixtures
}

\author{
Mina Bouhallaoui, Ali Benhra, Bouchra El Haimeur, Françoise Quiniou et \\ Mohammed Blaghen
}

Volume 30, numéro 3, 2017

Reçu le 26 mars 2015, accepté le 18 août 2016

URI : https://id.erudit.org/iderudit/1044245ar

DOI : https://doi.org/10.7202/1044245ar

\section{Aller au sommaire du numéro}

Éditeur(s)

Université du Québec - INRS-Eau, Terre et Environnement (INRS-ETE)

ISSN

1718-8598 (numérique)

Découvrir la revue

Citer cet article

Bouhallaoui, M., Benhra, A., El Haimeur, B., Quiniou, F. \& Blaghen, M. (2017). Utilisation du développement embryolarvaire de l'huitre creuse crassostrea gigas comme outil de diagnostic de la toxicité de substances pures et de mélanges complexes. Revue des sciences de l'eau / Journal of Water Science, 30(3), 171-181. https://doi.org/10.7202/1044245ar
Résumé de l'article

Les embryons et les larves des mollusques bivalves sont fréquemment utilisés comme organismes sentinelles dans l'évaluation de la qualité du milieu marin. En effet, ils sont très sensibles aux polluants et fournissent une réponse rapide. Le test d'embryotoxicité chez l'huître Crassostrea gigas a été utilisé pour évaluer la toxicité de trois métaux (mercure, cuivre, cadmium) et celle des rejets d'unités industrielles déversant directement dans le littoral atlantique Casablanca-Mohammedia (le rejet d'électrochimie et le rejet industriel mixte) et sur la côte d'El Jadida à $100 \mathrm{~km}$ de Casablanca (le rejet de traitement des phosphates). Après $24 \mathrm{~h}$ d'exposition aux milieux à tester, les effets exprimés sous forme de $\mathrm{CE}_{50}$ (concentration efficace qui a un effet de $50 \%$ ), ont été comparés. Les résultats des tests montrent que le mercure est le métal le plus toxique $\left(\mathrm{CE}_{50}=4,4 \mu \mathrm{g} \mathrm{Hg} \cdot \mathrm{L}^{-1}\right)$ suivi du cuivre $\left(\mathrm{CE}_{50}=16,4 \mu \mathrm{g} \mathrm{Cu} \cdot \mathrm{L}^{-1}\right.$ et enfin du cadmium $\left(\mathrm{CE}_{50}=46,9 \mu \mathrm{g} \mathrm{Cd} \cdot \mathrm{L}^{-1}\right)$. Les effets des effluents, testés à des concentrations croissantes, mettent en évidence un risque élevé de détérioration de la qualité du milieu marin, particulièrement dans les zones de déversement des rejets avec des $\mathrm{CE}_{50}$ atteintes avec moins de $1 \%$ de rejet dans le milieu, et permettent de classer ces effluents selon le gradient de toxicité croissant suivant : rejet d'électrochimie \&gt; rejet industriel mixte \&gt; rejet de traitement des phosphates. Cette étude nous a montré l'importance d'utilisation des embryons et larves d'huître comme outil sensible pour l'évaluation de la qualité du milieu. 


\section{UTILISATION DU DÉVELOPPEMENT EMBRYOLARVAIRE DE L'HUITRE CREUSE CRASSOSTREA GIGAS COMME OUTIL DE DIAGNOSTIC DE LA TOXICITÉ DE SUBSTANCES PURES ET DE MÉLANGES COMPLEXES}

Use of embryo-larval development stage of the gyster Crassostrea gigas as a toxicity assessment tool for pure substances and complex mixtures

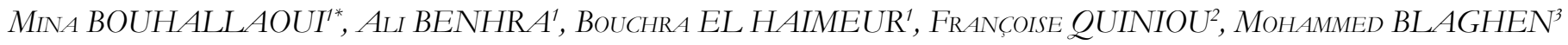

${ }^{1}$ Institut National de la Recherche Halieutique (INRH), 2, boulevard Sidi Abderrahmane, 20100 Casablanca, Maroc

${ }^{2}$ Institut Français de Recherche pour l'Exploitation de la Mer, 1625, route de Sainte-Anne, 29280 Plouzané, France

${ }^{3}$ Université Hassan II de Casablanca, Faculté des Sciences Aïn Chock, BP 5366, 20000 Casablanca, Maroc

Reçu le 26 mars 2015, accepté le 18 août 2016

\section{RÉSUMÉ}

Les embryons et les larves des mollusques bivalves sont fréquemment utilisés comme organismes sentinelles dans l'évaluation de la qualité du milieu marin. En effet, ils sont très sensibles aux polluants et fournissent une réponse rapide. Le test d'embryotoxicité chez l'huître Crassostrea gigas a été utilisé pour évaluer la toxicité de trois métaux (mercure, cuivre, cadmium) et celle des rejets d'unités industrielles déversant directement dans le littoral atlantique Casablanca-Mohammedia (le rejet d'électrochimie et le rejet industriel mixte) et sur la côte d'El Jadida à $100 \mathrm{~km}$ de Casablanca (le rejet de traitement des phosphates). Après $24 \mathrm{~h}$ d'exposition aux milieux à tester, les effets exprimés sous forme de $\mathrm{CE}_{50}$ (concentration efficace qui a un effet de $50 \%$ ), ont été comparés. Les résultats des tests montrent que le mercure est le métal le plus toxique $\left(\mathrm{CE}_{50}=\right.$
4,4 $\left.\mu \mathrm{g} \mathrm{Hg} \cdot \mathrm{L}^{-1}\right)$ suivi du cuivre $\left(\mathrm{CE}_{50}=16,4 \mu \mathrm{g} \mathrm{Cu} \cdot \mathrm{L}^{-1}\right.$ et enfin du cadmium $\left(\mathrm{CE}_{50}=46,9 \mu \mathrm{g} \mathrm{Cd} \cdot \mathrm{L}^{-1}\right)$. Les effets des effluents, testés à des concentrations croissantes, mettent en évidence un risque élevé de détérioration de la qualité du milieu marin, particulièrement dans les zones de déversement des rejets avec des $\mathrm{CE}_{50}$ atteintes avec moins de $1 \%$ de rejet dans le milieu, et permettent de classer ces effluents selon le gradient de toxicité croissant suivant : rejet d'électrochimie > rejet industriel mixte $>$ rejet de traitement des phosphates. Cette étude nous a montré l'importance d'utilisation des embryons et larves d'huître comme outil sensible pour l'évaluation de la qualité du milieu.

Mots-clés : Embryotoxicité, bio-essai, Crassostrea gigas, métaux, rejets industriels. 


\section{ABSTRACT}

Embryos and larva of bivalves are used frequently as sentinel organisms in ecotoxicological bioassays for the evaluation of seawater quality. Indeed, they are very sensitive to pollutants and provide a fast response. The embryo toxicity bioassay with the bivalve mollusc Crassostrea gigas was used to evaluate toxicity of three metals (mercury, copper, cadmium) and three effluents emanating from Moroccan industries which discharge directly on the Atlantic coast near Casablanca (electrochemical and mixed industrial waste waters) and on the El Jadida coast (phosphate treatment waste water). After 24-h exposures to the various media, the effects, expressed as $\mathrm{CE}_{50}$ values (effective concentrations affecting $50 \%$ of the test population), were compared. The results of the tests show that mercury is the most toxic metal $\left(\mathrm{CE}_{50}=4.4 \mu \mathrm{g} \mathrm{Hg} \cdot \mathrm{L}^{-1}\right)$ followed by copper $\left(\mathrm{CE}_{50}=16.4 \mu \mathrm{g} \mathrm{Cu} \cdot \mathrm{L}^{-1}\right)$ and finally cadmium $\left(\mathrm{CE}_{50}=\right.$ 46.9 $\left.\mu \mathrm{g} \mathrm{Cd} \cdot \mathrm{L}^{-1}\right)$. The effects of the industrial wastewaters, tested with increasing dilutions, indicate a strong risk of seawater quality deterioration, especially in the discharge zones with $\mathrm{CE}_{50}$ values corresponding to effluent concentrations of less than $1 \%$. The effluents decreased in toxicity according to the following order: electrochemical effluent > mixed industrial effluent $>$ phosphate treatment effluent. This study demonstrated the usefulness of oyster embryos and larvae as a sensitive tool for assessing environmental quality.

\section{Key Words: Embryo toxicity, bioassay, Crassostrea gigas, metals, industrial wastewaters.}

\section{INTRODUCTION}

Depuis de nombreuses années, les analyses chimiques de l'eau, du sédiment et des organismes donnent des indications sur la présence d'un polluant. Cependant, seules, ces indications sont insuffisantes et ne renseignent pas sur l'impact réel du polluant. Les effets biologiques des produits chimiques déversés dans le milieu naturel peuvent servir d'indicateurs de pollution, dans le règne animal et végétal, et permettent la mise en évidence précoce de contamination du milieu naturel avant l'altération de la structure des communautés. Les mollusques répondent pleinement aux différents critères pour le choix des organismes à retenir pour les bio-essais destinés à assurer une surveillance en zone littorale : vaste répartition géographique, possibilité d'utilisation toute l'année, intérêt économique (QUINIOU et TOULARASTEL, 1991; QUINIOU et al., 2005).

Comparées aux bivalves adultes (Crassostrea gigas et Mytilus galloprovincialis) retenues dans les études de surveillance, les larves de ces mêmes espèces présentent plusieurs avantages, notamment, une plus grande sensibilité vis-à-vis des métaux. De plus, les embryons et les larves ont un taux d'accumulation plus élevé en liaison avec leur développement rapide (RINGWOOD, 1990), ce qui permet de réaliser des observations de plus courtes durées. Le test d'embryotoxicité chez l'huître C. gigas recommandé par WOELKE (1972), a été sélectionné pour sa simplicité, sa rapidité et sa sensibilité, pour l'évaluation de la qualité biologique des eaux marines, estuariennes et des sédiments. Les études réalisées sur les effets toxiques des métaux sur les organismes marins ont surtout concerné les effets létaux et sublétaux sur les individus adultes maintenus dans des conditions expérimentales bien définies. Ces dernières années, il a été montré que de faibles concentrations d'éléments toxiques peuvent modifier le fonctionnement physiologique aussi bien du stade larvaire que celui adulte (HIS, 1991). L'objectif de ce travail est l'évaluation de la toxicité des métaux (mercure, cuivre et cadmium) et des rejets sur les stades de développement embryolarvaire de l'huître creuse $C$. gigas, après 24 h d'exposition.

\section{MATÉRIEL ET MÉTHODES}

Les essais ont été réalisés pendant la période naturelle de ponte des huîtres au Maroc (juin à septembre), avec des géniteurs provenant de la lagune d'Oualidia (Figure 1). Les organismes sont placés en stabulation en eau de mer à $20^{\circ} \mathrm{C}$ ou maintenus à sec jusqu'au moment de l'expérimentation en moins de 24 h. L'effet toxique des milieux a été évalué en déterminant le taux de larves $\mathrm{D}$ anormales, obtenu après incubation d'œufs fécondés durant $24 \mathrm{~h}$, selon le protocole de QUINIOU et al. (2005) et AFNOR (2009). Les bio-essais ont été réalisés avec l'eau de mer de la lagune, considérée comme non contaminée (CHEGGOUR et al., 1999), préfiltrée sur membrane de type Millipore (porosité de 0,22 $\mu \mathrm{m}$ ) et à une salinité de $30 \%$, compatible avec un bon développement embryonnaire des huittres (QUINIOU et al., 1997 et 2005; AFNOR, 2009). La salinité de l'eau de la lagune qui est située entre $34 \%$ et $35 \%$ a été ramenée à $30 \%$ par dilution avec de l'eau distillée.

La toxicité des trois effluents industriels (Figure 1) a été évaluée et comparée aux essais-témoins réalisés en eau de mer référence :

- Le rejet d'électrochimie (RE) émane d'une société d'électrochimie dont l'activité essentielle est la production du polychlorure de vinyle (PVC), du chlore et de la soude, faisant intervenir dans les procédés de production du mercure et du plomb.

- Le rejet industriel mixte (RIM) est un rejet complexe par lequel transitent des flux d'eaux domestiques et industrielles 


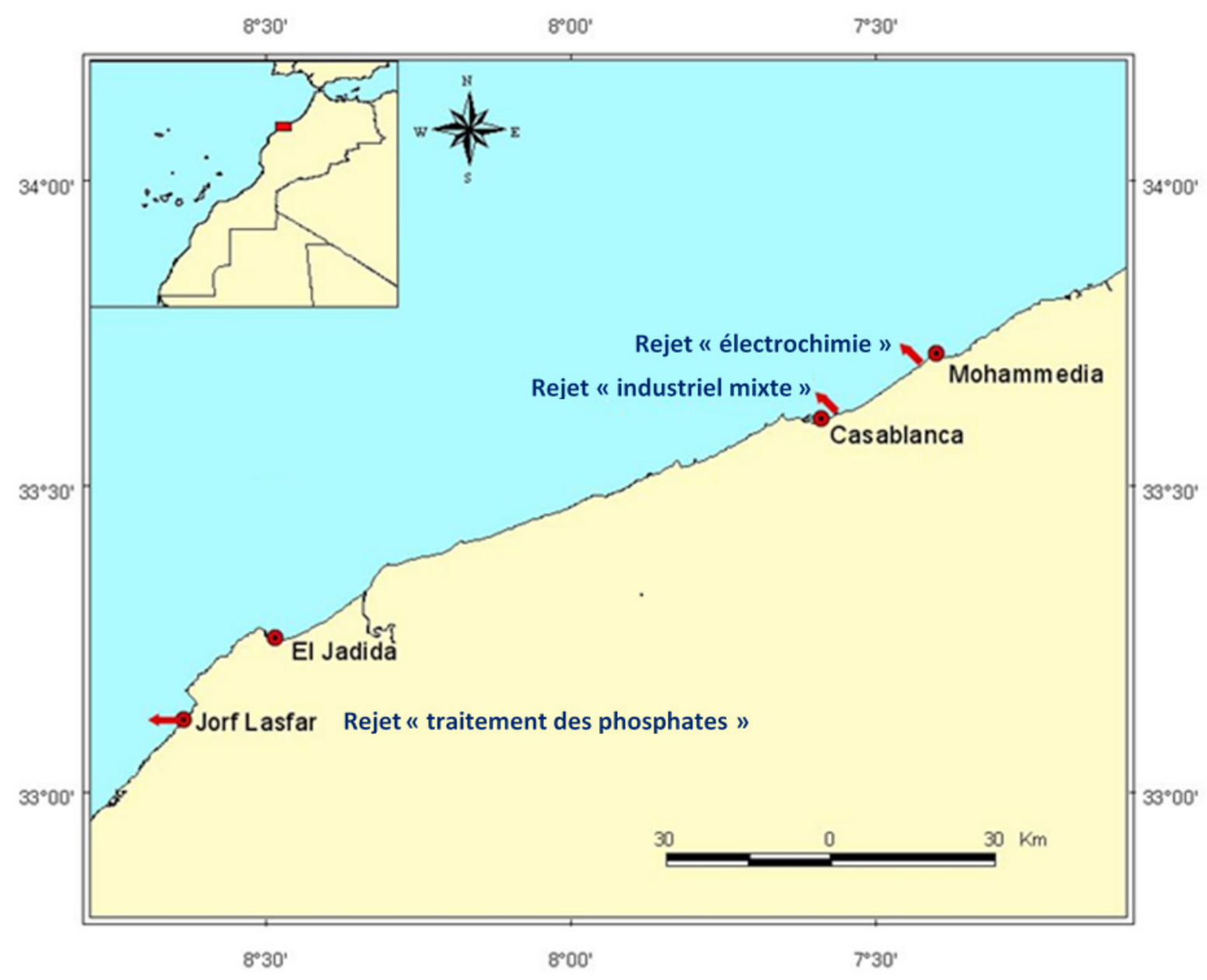

Figure 1. Localisation géographique des rejets étudiés. Map showing sources of studied sewage.

très hétérogènes, provenant principalement d'industries agroalimentaires et chimiques.

- Le rejet de traitement des phosphates (RTP) est issu du complexe chimique de Jorf Lasfar dont la fonction consiste en la production d'acide phosphorique par la transformation des phosphates et du soufre.

Les gammes de concentration des rejets obtenues par dilution dans l'eau de mer de référence, sont : 0,02\%, 0, 05\%, $0,2 \%, 0,5 \%$ et $1 \%$ pour RE, $0,1 \%, 0,5 \%, 1 \%, 2 \%$ et $5 \%$ pour RIM et $0,1 \%, 0,5 \%, 1 \%, 5 \%$ et $10 \%$ pour RTP.

Trois métaux (mercure, cuivre, cadmium) ont été également testés, sous forme de chlorures selon les gammes de concentrations suivantes (en $\left.\mu \mathrm{g} \cdot \mathrm{L}^{-1}\right)$ :

- $\mathrm{Cd}(\mathrm{II})\left(\mathrm{CdCl}_{2}, \mathrm{H}_{2} \mathrm{O}\right): 30-75-200-450-1000$

- $\mathrm{Cu}(\mathrm{II})\left(\mathrm{CuCl}_{2}, 2 \mathrm{H}_{2} \mathrm{O}\right): 20-50-120-300-800$

- $\mathrm{Hg}(\mathrm{II})\left(\mathrm{HgCl}_{2}\right): 10-25-60-150-400$

Le protocole du bio-essai utilisé est celui décrit par QUINIOU et al. (2005) et il correspond à une modification du protocole EPA (1996). Les essais ont été réalisés en triple dans des plaques Nunc multipuits stériles à raison de $3 \mathrm{~mL}$ par puits. Les œufs fécondés sont incubés dans les milieux à tester, à raison de 20000 à 50000 œufs par litre, pendant 24 h à $24^{\circ} \mathrm{C}$. À l'issue de cette période d'incubation, le développement est arrêté au stade larves $\mathrm{D}$ par addition du formol neutre à $8 \%$, et les pourcentages d'anomalies larvaires sont déterminés sur la base des critères de QUINIOU et TOULARASTEL (1991), HIS et al., (1999a), QUINIOU et al. (2005) et AFNOR (2009). Ces observations se font à l'aide d'un microscope inversé (grossissement 40x) sur 100 individus par réplicat. Les résultats sont exprimés en pourcentage d'anomalies larvaires. $\mathrm{La} \mathrm{CE}_{50}$, correspondant à la concentration entraînant 50 \% d'anomalies, est calculée à l'aide du logiciel Log Probit (EPA, 1991). Un test est considéré valide quand le pourcentage de larves anormales, enregistré au niveau des témoins, est inférieur à $20 \%$ (HIS et al., 1997; QUINIOU et al., 2005). La validité du test et la sensibilité des géniteurs sont également vérifiées en utilisant le sulfate de cuivre $\left(\mathrm{CuSO}_{4}, 5 \mathrm{H}_{2} \mathrm{O}\right)$ comme toxique de référence (QUINIOU et al., 2005). Les résultats sont exprimés en pourcentage brut de larves anormales. 


\section{RÉSULTATS}

\subsection{Validité du bio-essai}

Les taux d'anomalies larvaires pour les témoins sont inférieurs ou égaux à $10 \%$ lors de tous les tests réalisés. Par ailleurs, l'utilisation du toxique de référence $\left(\mathrm{CuSO}_{4}, 5 \mathrm{H}_{2} \mathrm{O}\right)$ a permis de vérifier la sensibilité des géniteurs. $\mathrm{La} \mathrm{CE}_{50}$ moyenne pour les huîtres marocaines est de $14 \mu \mathrm{g} \cdot \mathrm{L}^{-1} \mathrm{de} \mathrm{Cu}^{++}$. Ces résultats valident les bio-essais effectués dont les résultats peuvent donc être analysés.

\subsection{Effet des métaux}

Les pourcentages moyens d'anomalies larvaires, déterminés en fonction de la concentration du métal dans le milieu (Figure 2), montrent que le mercure exerce une grande toxicité sur le développement embryolarvaire de $C$. gigas. En effet, une concentration de $10 \mu \mathrm{g} \mathrm{Hg} \cdot \mathrm{L}^{-1}$ provoque en moyenne $85 \%$ d'anomalies larvaires. À partir de $60 \mu \mathrm{g} \cdot \mathrm{L}^{-1} \mathrm{du}$ métal dans le milieu, il n'y a plus de développement larvaire. Le cuivre présente lui aussi une grande toxicitévis-à-vis du développement larvaire; plus de $50 \%$ d'anomalies larvaires sont observées au niveau des pots contenant $20 \mu \mathrm{g} \cdot \mathrm{L}^{-1}$ seulement de cuivre. À $50 \mu \mathrm{g} \mathrm{Cu} \cdot \mathrm{L}^{-1}$, nous avons noté $90 \%$ d'anomalies avec un pourcentage important d'embryons.

Pour ce qui est du cadmium, sa toxicité embryolarvaire chez $C$. gigas reste inférieure à celles observées en présence du mercure et du cuivre (Figure 2). À une concentration de $30 \mu \mathrm{g} \cdot \mathrm{L}^{-1}$, le $\mathrm{Cd}$ provoque $45 \%$ d'anomalies dans le développement. À $1000 \mu \mathrm{g} \cdot \mathrm{L}^{-1}$, il n'y a plus de développement larvaire. La comparaison des $\mathrm{CE}_{50}$ des trois métaux testés (Tableau 1) montre que le mercure est le métal le plus toxique pour l'huître $C$. gigas $\left(\mathrm{CE}_{50}=4,4 \mu \mathrm{g} \cdot \mathrm{L}^{-1}\right)$ suivi du cuivre $\left(\mathrm{CE}_{50}=\right.$ $\left.16,4 \mu \mathrm{g} \cdot \mathrm{L}^{-1}\right)$ et du cadmium qui est le moins toxique avec une $\mathrm{CE}_{50}$ de $46,9 \mu \mathrm{g} \cdot \mathrm{L}^{-1}$.

Tableau 1. Concentrations efficaces $\left(\mathrm{CE}_{50}\right)$ de $\mathrm{Hg}(\mathrm{II}), \mathrm{Cd}(\mathrm{II})$ et $\mathrm{Cu}$ (II) avec les intervalles de confiance.

Table 1. Effective concentrations of $\mathrm{Hg}(\mathrm{II}), \mathrm{Cd}(\mathrm{II})$ and $\mathrm{Cu}(\mathrm{II})$ with confidence intervals.

\begin{tabular}{lcc}
\hline Métal & $\begin{array}{c}\mathbf{C E}_{50}-\mathbf{2 4} \mathbf{h} \\
\left(\boldsymbol{\mu g} \cdot \mathbf{L}^{-1}\right)\end{array}$ & Intervalle de confiance \\
\hline $\mathrm{Hg}(\mathrm{II})$ & 4,4 & $2,0-7,0$ \\
$\mathrm{Cu}(\mathrm{II})$ & 16,4 & $10,6-22,2$ \\
$\mathrm{Cd}(\mathrm{II})$ & 46,9 & $31,4-62,8$ \\
\hline
\end{tabular}

\subsection{Effet des effluents}

Les résultats des tests d'embryotoxicité réalisés sur les effluents issus de la zone côtière Casablanca-Mohammedia (RE, RIM) et celui du complexe phosphatier basé à Jorf Lasfar (RTP) sont représentés sur la figure 3. L'analyse de ces résultats montre l'existence d'une relation dose - effet. Le rejet de l'électrochimie (RE) exerce un grand effet toxique vis-à-vis des embryons et des larves de l'huître $C$. gigas. En effet, à 0,2 \% $(\mathrm{v} / \mathrm{v})$ de rejet, les taux d'anomalies larvaires dépassent $70 \%$, et avec la dilution de $1 \%$ du rejet dans le milieu, $80 \%$ d'individus sont bloqués au stade embryonnaire. La $\mathrm{CE}_{50}$ calculée est de $0,09 \%$ (Tableau 2).

Tableau 2. Concentrations efficaces $\left(\mathrm{CE}_{50}\right)$ des effluents avec les intervalles de confiance. $\mathrm{RE}$ : rejet d'électrochimie, RIM : rejet industriel mixte et RTP : rejet de traitement des phosphates.

Table 2. Effective concentrations of studied effluents with confidence intervals. Wastewater types: RE: electrochemical, RIM: mixed industrial, and RTP: phosphate treatment.

\begin{tabular}{lcc}
\hline Toxicité des effluents & $\begin{array}{c}\mathbf{C E}_{50}-\mathbf{2 4} \mathbf{h} \\
(\mathbf{\%} \mathbf{v} / \mathbf{v})\end{array}$ & $\begin{array}{c}\text { Intervalle de } \\
\text { confiance }\end{array}$ \\
\hline RE & 0,09 & $0,03-0,15$ \\
RIM & 0,16 & $0,08-0,23$ \\
RTP & 0,45 & $0,02-0,91$ \\
\hline
\end{tabular}

En ce qui concerne le rejet mixte (RIM), dès la plus faible dilution testée $(0,1 \%)$, il induit $59 \%$ d'anomalies larvaires. La toxicité augmente progressivement avec la concentration pour atteindre $100 \%$ d'anomalies à $2 \%$ de rejet; la $\mathrm{CE}_{50}$ est de $0,16 \%$.

La toxicité du rejet de traitement des phosphates (RTP) se manifeste également dès la dilution $0,1 \%$ avec $44 \%$ d'anomalies larvaires. Toutefois, ce rejet a un effet toxique moins drastique que RE et RIM; la $\mathrm{CE}_{50}$ calculée pour ce rejet est de $0,45 \%$.

\section{DISCUSSION ET CONCLUSION}

La validité du bio-essai a été vérifiée, en particulier par l'utilisation d'un témoin positif $\left(\mathrm{CuSO}_{4}, 5 \mathrm{H}_{2} \mathrm{O}\right)$. $\mathrm{La} \mathrm{CE}_{50}$ moyenne pour les huîtres marocaines est de $14 \mu \mathrm{g} \mathrm{Cu} \cdot \mathrm{L}^{-1}$, ce qui est en accord avec la littérature (Tableau 3). 
$\mathrm{Hg}$

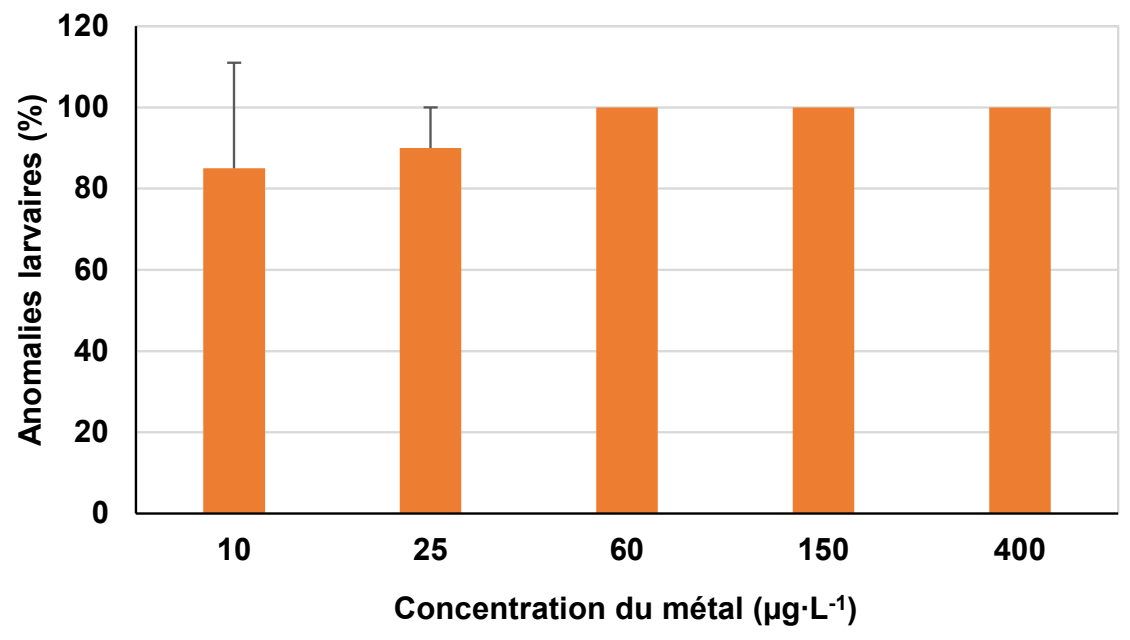

$\mathrm{Cu}$

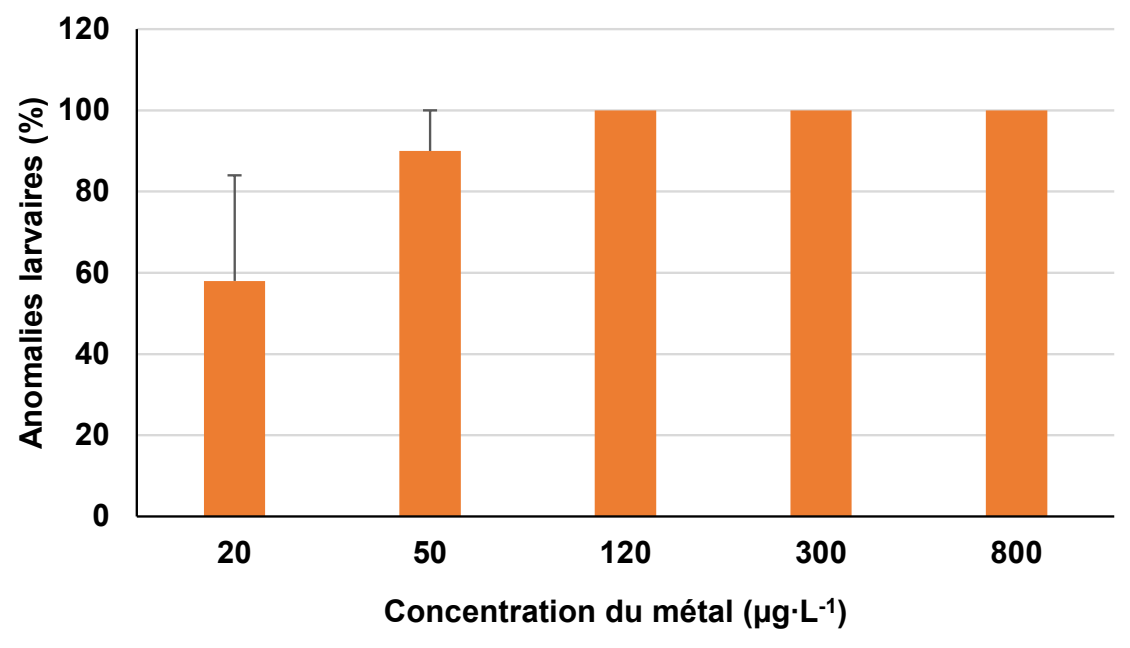

Cd

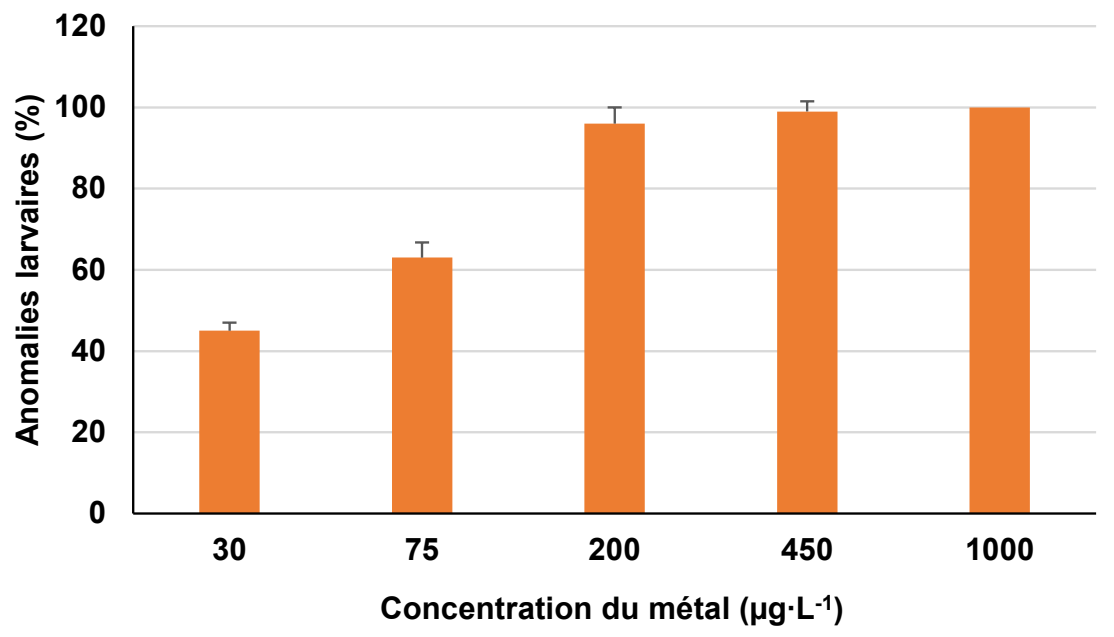

Figure 2. Toxicité du mercure $(\mathrm{Hg})$, du cuivre $(\mathrm{Cu})$ et du cadmium $(\mathrm{Cd})$ sur le développement embryolarvaire de Crassostrea gigas. Résultats exprimés en pourcentages d'anomalies larvaires \pm écart-type $(n=3)$.

Toxicity of mercury $(\mathrm{Hg})$, copper $(\mathrm{Cu})$, and cadmium (Cd) on embryo-larval development of C. gigas. Results expressed as percentages of larval anomalies $\pm S D(n=3)$. 
RE
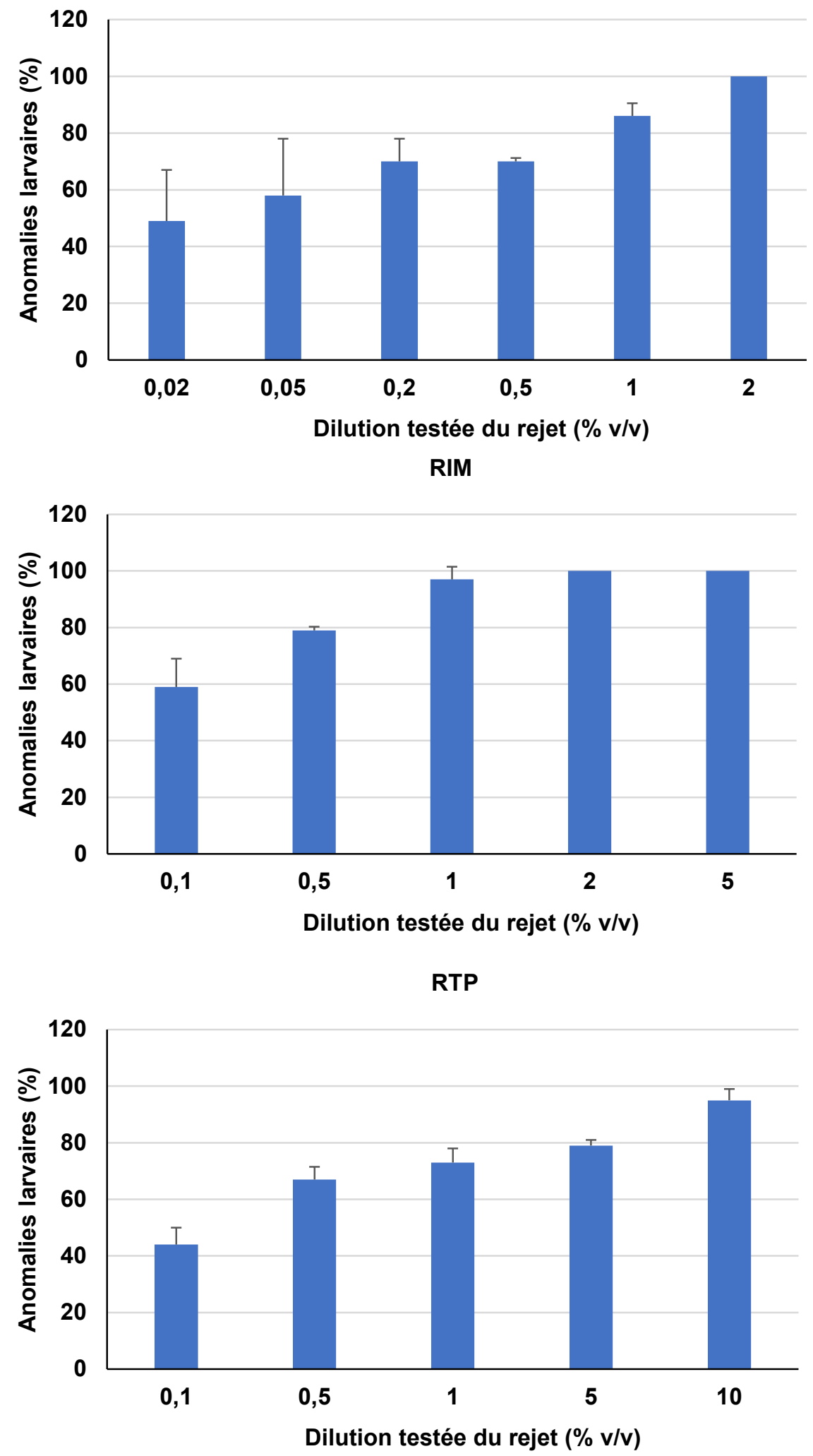

Figure 3. Toxicité des trois rejets industriels testés (RE : rejet d'électrochimie, RIM : rejet industriel mixte, RTP : rejet de traitement des phosphates). Résultats exprimés en pourcentages d'anomalies larvaires \pm écart-type $(n=3)$.

Toxicity of three industrial wastewaters tested (RE: electrochemical, RIM: mixed industrial, RTP: phosphate treatment). Results expressed as percentages of larval anomalies $\pm S D(n=3)$. 
Tableau 3. Comparaison des concentrations efficaces $\left(\mathrm{CE}_{50}\right)$ pour le sulfate de cuivre $\left(\mathrm{CuSO}_{4}, 5 \mathrm{H}_{2} \mathrm{O}\right)$ testé chez Crassostrea gigas.

Table 3. Comparison of $\mathrm{EC}_{50}$ for copper sulfate $\left(\mathrm{CuSO}_{4}\right.$ $5 \mathrm{H}_{2} \mathrm{O}$ ) tested in $\mathrm{C}$. gigas.

\begin{tabular}{lc}
\hline Référence de l'étude & $\begin{array}{c}\mathbf{C E}_{50}-\mathbf{2 4} \mathbf{~ h} \\
\left(\boldsymbol{\mu g ~} \mathbf{C u}^{++} \cdot \mathbf{L}^{-1}\right)\end{array}$ \\
\hline Présente étude & 14 \\
GEFFARD, 2001 & 14,7 \\
BRETON, 1999 & 12,3 \\
AFNOR ISO, 2015 & $10,95 \pm 3,10$ \\
COGLIANESE, 1982 & 10 \\
HIS et ROBERT, 1982 & 13 \\
\hline
\end{tabular}

\subsection{Toxicité des métaux}

Les résultats obtenus montrent que la phase de développement embryolarvaire de $C$. gigas est très sensible à l'action des métaux; des anomalies de développement sont enregistrées à des concentrations sublétales très inférieures aux doses létales. Les $\mathrm{CE}_{50}$ calculées pour les trois métaux $(\mathrm{Hg}$, $\mathrm{Cu}, \mathrm{Hg}$ ) sont faibles. En effet, après le tributylétain (TBT), les métaux exercent une toxicité plus élevée qui est de l'ordre de quelques microgrammes par litre par rapport aux autres polluants tels que les pesticides, dont l'action défavorable est de l'ordre de plusieurs centaines de microgrammes par litre (HIS, 1991). Les réponses sublétales des larves (croissance, anomalies, etc.) aux métaux sont généralement des critères plus sensibles que la mortalité larvaire (HIS et al., 1999a).

Le cuivre est un métal dont la toxicité à différents niveaux de l'organisation (mortalité, comportement, embryogenèse) chez les bivalves n'est plus à démontrer; ce métal exerce une toxicité sur le développement embryolarvaire chez C. gigas, dont l'importance vient après celle du mercure $\left(\mathrm{CE}_{50}-24 \mathrm{~h}=\right.$ $16,4 \mu \mathrm{g} \cdot \mathrm{L}^{-1}$ ). En effet, les sels de ce métal (oxyde de $\mathrm{Cu}$, sulfate de $\mathrm{Cu}$ et chlorure de $\mathrm{Cu}$ ) entrent dans la composition des peintures antisalissures qui perturbent profondément les activités aquacoles. HIS (1991), en étudiant l'effet de ces différents sels sur le développement embryolarvaire de l'huître $C$. gigas, a montré que le chlorure de cuivre provoque une inhibition du développement larvaire à $50 \mu \mathrm{g} \cdot \mathrm{L}^{-1}$ et la croissance larvaire est nettement retardée jusquà la concentration de $10 \mu \mathrm{g} \cdot \mathrm{L}^{-1}$. La comparaison de nos résultats concernant la toxicité du cuivre sur le développement avec ceux de la littérature montre que les valeurs de $\mathrm{CE}_{50}$ sont faibles; HIS et al. (1999b) ont calculé une $\mathrm{CE}_{50}$ plus élevée chez la même espèce $\left(37 \mu \mathrm{g} \cdot \mathrm{L}^{-1}\right)$. MAI et al. (2012) ont enregistré une embryotoxicité du cuivre chez l'huître japonaise à partir d'une concentration de $0,1 \mu \mathrm{g} \cdot \mathrm{L}^{-1}$ dans le milieu, avec une $\mathrm{CE}_{50}$ encore plus faible que celle que nous avons calculée; la même étude a révélé une forte corrélation entre le pourcentage d'anomalies larvaires et celui des dommages d'ADN observés suite à une exposition au cuivre.

La $\mathrm{CE}_{50}-48 \mathrm{~h}$ déterminée par RAMACHANDRAN et al. (1997) chez l'oursin de mer Diadema setosum pour la larve Pluteus est de $40 \mu \mathrm{g} \cdot \mathrm{L}^{-1}$; ces auteurs ont postulé que le cuivre était plus toxique que le cadmium sur la fertilisation des gamètes de l'oursin. Par ailleurs, il a été montré que le cuivre provoque la réduction de la croissance chez les juvéniles du bivalve Scrobicularia plana à une concentration de $10 \mu \mathrm{g} \cdot \mathrm{L}^{-1}$ (RUIZ et al., 1994).

Lecadmium exerceluiaussiune toxicitésur le développement larvaire de $C$. gigas. La $\mathrm{CE}_{50}-24$ h est estimée à $46,9 \mu \mathrm{g} \mathrm{Cd} \cdot \mathrm{L}^{-1}$. L'effet de ce métal reste toutefois faible en comparaison avec celui du mercure et du cuivre sur le développement embryolarvaire. RINGWOOD (1990) a postulé l'existence d'une adaptation physiologique des véligères du bivalve Isognomon californicum à l'exposition au cadmium; cette résistance serait imputée selon cet auteur à l'induction des métallothionéines. CALABRESE et al. (1973) ont également montré que le cadmium était le moins toxique vis-à-vis des embryons de Crassostrea virginica par rapport aux autres métaux $(\mathrm{Hg}, \mathrm{Cu}, \mathrm{Zn})$ avec une $\mathrm{CL}_{50}$ plus élevée de $10,3 \mu \mathrm{g} \cdot \mathrm{L}^{-1}$. Une autre étude a montré que le $\mathrm{Cd}$ exerce un effet sur le développement embryolarvaire de l'huître C. gigas trois fois supérieur au contrôle, mais qui reste inférieur à celui enregistré lors de la présente étude, avec une $\mathrm{CE}_{50}$ plus élevée de $212 \mu \mathrm{g} \cdot \mathrm{L}^{-1}$ (MAI et al., 2012). Par ailleurs, une expérimentation réalisée sur les effets de $\mathrm{Cd}$ et de $\mathrm{Hg}$ sur le comportement larvaire de Eurypanopeus depressus a montré que $\mathrm{Cd}$ à des concentrations sublétales perturbe l'activité de nage des larves avec une augmentation du taux de mortalité, mais son effet ne s'exerce pas à tous les stades, comparé au mercure dont la toxicité s'observe à différents stades de la vie larvaire de cette espèce (MIRKES et al., 1978).

Les résultats de l'embryotoxicité des métaux ont montré que le mercure exerce une grande toxicité sur le développement larvaire de $C$. gigas $\left(\mathrm{CE}_{50}-24 \mathrm{~h}=4,4 \mu \mathrm{g} \cdot \mathrm{L}^{-1}\right)$, et la majorité des anomalies correspondent à des embryons qui n'ont pas atteint le stade coquillier. Ce métal est en effet classé en tête de liste de toxicité des métaux sur les premiers stades de développement chez les bivalves (HIS et al., 1999a). BEIRAS et HIS (1994 et 1995) ont trouvé que l'embryogenèse était anormale pour $50 \%$ des individus à $11 \mu \mathrm{g} \mathrm{Hg} \cdot \mathrm{L}^{-1}$ chez $C$. gigas. Ces études ont montré que la larve $\mathrm{D}$ est un stade très sensible au mercure; les $\mathrm{CL}_{50}$ augmentent en passant du stade larve $\mathrm{D}$, la larve umbonée à la pédivéligère (de 33 à $200 \mu \mathrm{g} \mathrm{Hg} \cdot \mathrm{L}^{-1}$ ) pour la même espèce. Une étude comparative entre les bio-essais larvaires chez C. gigas et l'oursin Paracentrotus lividus a montré que l'effet du mercure sur le développement de Crassostrea était plus significatif, avec une $\mathrm{CE}_{50}$ plus faible que celle enregistrée chez l'oursin (HIS et al., 1999b). CALABRESE et al. (1973) ont également montré que les stades embryons, et à moindre degré les larves, 
de $C$. virginica sont plus sensibles au mercure que les stades adultes, et ont relevé une $\mathrm{CL}_{50}-48 \mathrm{~h}$ de $5,6 \mu \mathrm{g} \mathrm{Hg} \cdot \mathrm{L}^{-1}$. Toutefois, la métamorphose chez les bivalves est plus résistante au mercure par rapport aux autres stades larvaires; la concentration efficace qui réduit la métamorphose chez $50 \%$ des individus exposés est de $60 \mu \mathrm{g} \cdot \mathrm{L}^{-1}$, alors que la croissance larvaire est réduite à une concentration de $4 \mu \mathrm{g} \mathrm{Hg} \cdot \mathrm{L}^{-1}$ (BEIRAS et HIS, 1994).

L'estimation de la toxicité potentielle des métaux au moyen du bio-essai "embryotoxicité " a permis d'établir un gradient de toxicité identique à celui trouvé par le test de létalité réalisé avec C. gigas à savoir : $\mathrm{Hg}$ plus toxique que $\mathrm{Cu}$ qui est plus toxique que Cd (BOUHALLAOUI et al., 2003). BELLAS et al. (2001) ont trouvé le même gradient de toxicité métallique chez Ciona intestinalis, avec des $\mathrm{CE}_{50}$ supérieures à celles enregistrées chez C. gigas.

\subsection{Toxicité des effluents}

Les résultats obtenus dans l'évaluation de l'impact des rejets industriels sur le développement embryolarvaire de l'huître creuse $C$. gigas à travers le test d'embryotoxicité ont montré que les embryons et les larves sont très sensibles aux rejets, puisque nous observons des effets néfastes à des concentrations aussi faibles que 0,02\% du rejet dans le milieu (cas du rejet d'électrochimie). L'analyse de ces résultats a montré que les trois rejets sont toxiques sur le développement larvaire de $C$. gigas.

La comparaison des résultats avec les différentes études sur les stades larvaires de bivalves est limitée par la différence dans le temps d'exposition d'une étude à l'autre, les conditions d'incubation des larves, le paramètre observé et la différence dans la taille; les larves de grande taille sont supposées être plus résistantes aux polluants (BEIRAS et HIS, 1995).

L'huître C. gigas présente une grande sensibilité aux rejets en comparaison avec la moule $M$. galloprovincialis; les $\mathrm{CE}_{50}-24 \mathrm{~h}$ enregistrées chez l'huître pour le rejet mixte et celui d'électrochimie $(0,16 \%$ et $0,09 \%$ respectivement) sont largement inférieures aux $\mathrm{CE}_{50}-48 \mathrm{~h}$ déterminées chez la moule $(2,61 \%$ pour le rejet mixte et $2,23 \%$ pour le rejet d'électrochimie) (BOUHALLAOUI et al., 2011). L'évaluation de la toxicité de ces mêmes rejets à travers le bio-essai d'embryotoxicité utilisant les anomalies larvaires chez l'oursin de mer Paracentrotus lividus a montré le même profil d'impact, avec des $\mathrm{CE}_{50}-48 \mathrm{~h}$ de 2,08\% pour RE, et de $2,56 \%$ pour le RIM (EL HAIMEUR et al., 2013). Ces valeurs de $\mathrm{CE}_{50}-48 \mathrm{~h}$ restent toutefois, supérieures à celles enregistrées chez C. gigas, ce qui confirme la grande sensibilité des bio-essais sur les stades larvaires de l'huître.
$\mathrm{Si}$ l'on cherche à comparer les valeurs de $\mathrm{CE}_{50}$ (embryotoxicité) avec les valeurs de $\mathrm{CL}_{50}$ (toxicité létale chez C. gigas) déterminées pour ces mêmes rejets par le bio-essai de létalité (BOUHALLAOUI et al., 2003), il se confirme que le paramètre embryotoxicité (anomalies larve D) est beaucoup plus sensible aux effluents $\left(\mathrm{CE}_{50}-24 \mathrm{~h}=0,09 \%\right.$ pour $\left.\mathrm{RE}\right)$ que le critère de mortalité chez le même bivalve $\left(\mathrm{CL}_{50}-96 \mathrm{~h}=\right.$ 16,6 \% pour RE), avec le même profil de toxicité. La sensibilité des différents stades de développement aux toxiques varie en fonction de l'âge; selon AMIARD-TRIQUET (1983), il existe généralement un facteur de 30 entre le stade gamètes et le stade adulte, le plus résistant. Ceci est en parfait accord avec le constat relevé par plusieurs auteurs postulant que les stades précoces de développement embryonnaire sont plus sensibles aux substances toxiques que les individus adultes (CALABRESE et al., 1973; ROBERT et al., 1986; QUINIOU et TOULARASTEL, 1991; HIS et SEAMAN, 1993; BEIRAS et HIS, 1994 et 1995; QUINIOU et al.,1997; HIS et al.,1997; GEFFARD, 2001).

Les paramètres physicochimiques de ces rejets (Tableau 4) révèlent une charge polluante intense, surtout les chlorures (BOUTHIR et al., 2004) avec des niveaux qui dépassent les valeurs admises dans le cas de l'effluent d'électrochimie, et le caractère basique $(\mathrm{pH}=11)$ de ce rejet explique également sa grande toxicité. Par ailleurs, la charge métallique $(\mathrm{Cd}, \mathrm{Pb}, \mathrm{Hg}$ et $\mathrm{Cu}$ ) des moules prélevées aux abords du rejet $\mathrm{RE}$ révèle une contamination importante en mercure dépassant parfois les normes admises (allant jusqu'à $7,7 \mu \mathrm{g} \cdot \mathrm{g}^{-1}$ poids sec) avec $20 \%$ du méthylmercure, qui est la forme la plus toxique (CHAFIK et al., 2001; INRH, 2002). L'effet toxique élevé de ce rejet serait à relier à la présence de mercure qui est utilisé, lors des procédés de production effectués par cette société.

L'effluent industriel mixte accuse des valeurs très élevées en matière organique, en matière en suspension $\left(988 \mathrm{mg} \cdot \mathrm{L}^{-1}\right)$ et en éléments nutritifs, surtout les orthophosphates et les sulfates (Tableau 4). Il présente des valeurs de la demande chimique en oxygène $\left(\mathrm{DCO}=2180 \mathrm{mg} \cdot \mathrm{L}^{-1}\right)$ qui se traduisent par un apport important de la matière organique. Les teneurs métalliques $(\mathrm{Cu}$, $\mathrm{Cd}$ ) enregistrées au niveau du rejet industriel mixte montrent que ce rejet est chargé en métaux, en particulier le $\mathrm{Cu}$ avec une concentration qui dépasse $200 \mu \mathrm{g} \cdot \mathrm{L}^{-1}$ (BOUTHIR et al., 2004), ce qui explique sa toxicité sur le développement larvaire de ce bivalve.

Le rejet de traitement des phosphates exerce lui aussi un effet sur le développement embryolarvaire de C. gigas. La qualité physicochimique (Tableau 4) des eaux du collecteur principal de RTP montre aussi des dépassements au niveau des phosphates $\left(55 \mathrm{mg} \cdot \mathrm{L}^{-1}\right)$, témoignant d'un apport considérable par cet émissaire, pouvant conduire à l'eutrophisation du milieu littoral récepteur. La toxicité du RTP serait également due à sa 
Tableau 4. Qualité physicochimique moyenne des rejets testés (présente étude; BOUTHIR et al., 2004; KAIMOUSSI, 2002 ).

Table 4. Average of physico chemical quality of industrial wastewaters tested

\begin{tabular}{|c|c|c|c|c|c|c|c|c|c|c|c|}
\hline $\begin{array}{l}\text { Toxicité } \\
\text { des effluents }\end{array}$ & $\begin{array}{c}t \\
\left({ }^{\circ} \mathrm{C}\right)\end{array}$ & $\mathrm{pH}$ & $\begin{array}{c}\text { Salinité } \\
(\% 0)\end{array}$ & $\begin{array}{c}\mathrm{MES}^{\mathrm{a}} \\
\left(\mathrm{mg} \cdot \mathrm{L}^{-1}\right)\end{array}$ & $\begin{array}{c}\mathrm{NO}_{3}^{-} \\
\left(\mathrm{mg} \cdot \mathrm{L}^{-1}\right)\end{array}$ & $\begin{array}{c}\mathrm{NO}_{2}^{-} \\
\left(\mathrm{mg} \cdot \mathrm{L}^{-1}\right)\end{array}$ & $\begin{array}{c}\mathrm{NH}_{4}^{+} \\
\left(\mathrm{mg} \cdot \mathbf{L}^{-1}\right)\end{array}$ & $\begin{array}{c}\mathrm{PO}_{4}^{3-} \\
\left(\mathrm{mg} \cdot \mathrm{L}^{-1}\right)\end{array}$ & $\begin{array}{c}\mathrm{SO}_{4}{ }^{2-} \\
\left(\mathrm{mg} \cdot \mathrm{L}^{-1}\right)\end{array}$ & $\begin{array}{c}\mathrm{Cl}^{-} \\
\left(\mathrm{mg} \cdot \mathrm{L}^{-1}\right)\end{array}$ & $\begin{array}{c}\mathrm{DCO}^{\mathrm{b}} \\
\left(\mathrm{mg} \cdot \mathrm{L}^{-1}\right)\end{array}$ \\
\hline $\mathrm{RE}^{\mathrm{c}}$ & 27,3 & 11,7 & 16,2 & 127,2 & 1,48 & 0,04 & 15,6 & 4,69 & 265 & 3190 & 1424 \\
\hline RIM $^{\mathrm{d}}$ & 32,9 & 8,05 & 8,8 & 988,4 & 0,26 & 0,05 & 39,95 & 165,8 & 3151 & 1718 & 2180 \\
\hline $\mathrm{RTP}^{\mathrm{e}}$ & 27,5 & 1,9 & - & 1069 & - & - & 8,7 & 55 & 7241 & 21,3 & 765 \\
\hline
\end{tabular}

nature acide $(\mathrm{pH}=2)$. Une étude réalisée sur l'évaluation de l'impact des rejets de l'industrie des phosphates installée à Jorf Lasfar sur le milieu a montré que les moules de cette région sont très contaminées par le $\mathrm{Cd}$ et le $\mathrm{Cu}$, particulièrement au niveau des points du rejet; ces métaux sont originaires des résidus phosphatiers (phosphogypse) déversés dans l'eau de mer (CHEGGOUR et al., 2000). KAIMOUSSI (2002) a en effet détecté des teneurs élevées en métaux lourds au niveau du collecteur principal du RTP, particulièrement le cuivre (Tableau 5). Ces polluants se manifestent au niveau des organismes marins et provoquent ainsi la dégradation du milieu.

NELSON etal. (1983) ont mis en évidence des perturbations dans le développement larvaire de $C$. virginica imputables à l'action des eaux de ruissellement; ils ont démontré l'existence d'une toxicité résiduelle un mois après le déversement dans la zone de rejet. Par ailleurs, les mélanges métalliques incluant le mercure en particulier sont plus toxiques sur les embryons de bivalves que les éléments métalliques individuels (HIS et al., 1996).

Les résultats recueillis dans cette étude mettent en évidence le danger que peuvent constituer les activités anthropiques pour les écosystèmes littoraux, notamment une détérioration des conditions de milieu, particulièrement dans les zones de déversement de rejets dont la composition chimique en polluants a des proportions parfois variables, est souvent inconnue. Les $\mathrm{CE}_{50}$ enregistrées témoignent de la grande nocivité des effluents déversés dans le milieu marin sans traitement préalable.

En conclusion, ces résultats confirment que les larves de Crassostrea gigas constituent un matériel de choix pour la surveillance de la qualité du milieu marin. En effet, les tests basés sur l'utilisation des larves de $C$. gigas permettent de déceler la présence de substances toxiques dans l'eau, lorsque les méthodes de la chimie analytique ne sont pas assez sensibles.
Tableau 5. Concentrations métalliques moyennes au niveau du collecteur principal du RTP (rejet de traitement des phosphates) (KAIMOUSSI, 2002).

Table 5. Average of metal concentrations in the RTP (phosphate treatment wastewaters) collector.

\begin{tabular}{lc}
\hline Métaux & $\begin{array}{c}\text { Collecteur RTP } \\
\left(\boldsymbol{\mu g} \cdot \mathbf{L}^{-1}\right)\end{array}$ \\
\hline $\mathrm{Cd}$ & 52,5 \\
$\mathrm{Cu}$ & 349 \\
$\mathrm{Fe}$ & 3100 \\
$\mathrm{Zn}$ & 15000 \\
\hline
\end{tabular}

\section{RÉFÉRENCES BIBLIOGRAPHIQUES}

\section{ASSOCIATION FRANÇAISE DE NORMALISATION} (AFNOR) (2009). Bio-indicateur de la toxicité potentielle de milieu aqueux - Détermination de la toxicité potentielle d'échantillons aqueux sur le développement embryo-larvaire de bivalve. XP-T90-382. 684 AFNOR, La Plaine-SaintDenis, Paris, France, 18 p.

ASSOCIATION FRANÇAISE DE NORMALISATION (AFNOR) (2015). Qualité de l'eau - Détermination de la toxicité d'échantillons aqueux sur le développement embryolarvaire de l'huître creuse (Crassostrea gigas) et de la moule (Mytilus edulis ou Mytilus galloprovincialis). AFNOR, ISO 17244:2015, Paris, France, 24 p.

AMIARD-TRIQUET C. (1983). Les tests de toxicité aiguë en milieu aquatique : méthodologie, standardisation, interprétation, limites d'application. Océanis, 9, 451-463.

BEIRAS R. et E. HIS (1994). Effects of dissolved mercury on embryogenesis, survival growth and metamorphosis of Crassostrea gigas oyster larvae. Mar. Ecol. Prog. Ser., 113, 95-103. 
BEIRAS R. et E. HIS (1995). Effects of dissolved mercury on embryogenesis, survival and growth of Mytilus galloprovincialis mussel larvae. Mar. Ecol. Prog. Ser., 126, 185-189.

BELLAS J., E. VAZQUEZ et R. BEIRAS (2001). Toxicity of $\mathrm{Hg}, \mathrm{Cu}, \mathrm{Cd}$ and $\mathrm{Cr}$ on early developmental stages of Ciona intestinalis (Chordata, Ascidiacea) with potential application in marine water quality assessment. Water Res., 35 (12), 2905-12.

BOUHALLAOUI M., A. BENHRA, A. CHAFIK et M. BLAGHEN (2003). L'huître creuse $C$. gigas, un outil de biodétection de la pollution marine. Bull. Soc. Zool. Fr., $128,35-48$.

BOUHALLAOUI M., A. BENHRA, H. NILI, B. EL HAIMEUR, S. EL AMRANI et A. CHAFIK (2011). Utilisation des stades de développement embryolarvaire chez Mytilus galloprovincialis pour l'évaluation de la qualité des eaux marines et de la toxicité des rejets. Bull. Soc. Zool. Fr., 136, 205-218.

BOUTHIR F.Z., A. CHAFIK, S. BENBRAHIM, S. SOUABI, H. El MERDHY, A. MESSOUDI et M. SIFEDDINE (2004). Qualité physico-chimique des eaux côtières du littoral de la wilaya du grand Casablanca (Océan Atlantique marocain) utilisant la moule Mytilus galloprovincialis comme indicateur de la contamination métallique. Mar. Life, 14, 59-70.

BRETON A. (1999). Évaluation de la sensibilité de lots d'huîtres creuses envers un toxique de référence, le sulfate de cuivre, lors de bio-essais sur le développement embryonnaire. Rapport de stage. Univ. de Metz, France, 92 p.

CALABRESE A., R.S. COLLIER, D.A. NELSON et J.R. MCINNES (1973). The toxicity of heavy metals to embryos of the American oyster C. virginica. Mar. Biol., $18,162-166$.

CHAFIKA., M. CHEGGOUR, D. COSSA, S. BENBRAHIM et M. SIFEDDINE (2001). Quality of Moroccan Atlantic coastal waters: water monitoring and mussel watching. Aquat. Living Resour., 14, 239-249.

CHEGGOUR M., A. CHAFIK, H. TEXIER, A.BOUHALLAOUI, N.GHARBI,A.BOUMEZZOUGH et J. ELATTAR (1999). Bioaccumulation de quelques éléments métalliques chez l'huître Crassostrea gigas (Thunberg) en élevage dans la lagune de Oualidia (Maroc) : Rôle des facteurs écologiques et biologiques. Haliotis, 28, 31-44.
CHEGGOUR M., W.J. LANGSTON, A. CHAFIK, H. TEXIER, H. IDRISSI et A. BOUMEZZOUGH (2000). Phosphate industry discharges and their impact on metal contamination and intertidal macrobenthos: Jorf Lasfar and Safi coastlines (Morocco). Toxicol. Environ. Chem., 70, 159-179.

COGLIANESE M.P. (1982). The effects on salinity on copper and silver toxicity to embryos of the Pacific oyster. Arch. Environ. Contam. Toxicol., 11, 297-303.

EL HAIMEUR B., M. BOUHALLAOUI, S. FARAH, H. ELMORTAJI et A. BENHRA (2013). Utilisation des stades de développement embryo-larvaire chez l'oursin de mer Paracentrotus lividus pour l'évaluation de la qualité des eaux marines et de la toxicité des rejets. Bull. Soc. Zool. Fr., $138,165-176$.

ENVIRONMENTAL PROTECTION AGENCY (EPA) (1991). Methods for measuring the acute toxicity of effluents and receiving waters to freshwater and marine organisms. EPA 600/4-90-027, Cincinnati, OH, États-Unis, 275 p.

ENVIRONMENTAL PROTECTION AGENCY (EPA) (1996). Ecological effects test guidelines. Bivalves acute toxicity test (embryo-larval). EPA/OPPTS 850-1055, Cincinnati, $\mathrm{OH}$, États-Unis, 7 p.

GEFFARD O. (2001). Toxicité potentielle des sédiments marins et estuariens contaminés : évaluation chimique et biologique, biodisponibilité des contaminants sédimentaires. Thèse de doctorat, Univ. Bordeaux 1, France, 351 p.

HIS E. (1991). Biologie et écotoxicologie des véligères de Crassostrea gigas (Thunberg) dans le bassin d'Arcachon. Thèse de Doctorat, Univ. Bordeaux 1, France, 192 p.

HIS E. et R. ROBERT (1982). Le danger des traitements par le sulfate de cuivre en zone conchylicole : toxicité vis-à-vis des œufs et des jeunes larves de C. gigas. Rev. Trav. Inst. Pêches Marit., 42 (2), 117-125.

HIS E. et M.N.L. SEAMAN (1993). Effects of twelve pesticides on larvae of oysters (C. gigas) and two species of unicellular marine algae (Isochrysis galbana and Chaetoceros calcitrans). International Council for the Exploration of the Sea, ICES-CM, Copenhague, Danemark, 8 p.

HIS E., R. BEIRAS, M.N.L. SEAMAN, N.M. TRIEFF et G. PAGANO (1996). Sublethal and lethal toxicity of aluminium industry effluent to early development stage of the C. gigas oyster. Arch. Environ. Contam. Tox., 30, 335339 . 
HIS E., M.N.L. SEAMAN et R. BEIRAS (1997). A simplification of the bivalve embryogenesis larval development bioassay method for water quality assessment. Water Res., 31, 351-355.

HIS E., R. BEIRAS et SEAMAN M.N.L. (1999a). The assessment of marine pollution: bioassays with bivalve embryos and larvae. Adv. Mar. Biol., 37, 1-178.

HIS E., I. HEYVANG, O. GEFFARD et X. DE MONTAUDOUIN (1999b). A comparison between oyster (Crassostrea gigas) and sea urchin (Paracentrotus lividus) larval bioassays for toxicological studies. Water Res., 33, 1706-1718.

INSTITUT NATIONAL DE LA RECHERCHE HALIEUTIQUE (INRH) (2002). Évaluation de la qualité et de la salubrité du milieu marin. Rapport annuel de l'état de l'environnement marin, 1997-2001, Casablanca, Maroc, $100 \mathrm{p}$.

KAIMOUSSI A. (2002). État de la pollution métallique au niveau du littoral de la région d'El Jadida : étude comparative entre l'année 94/95 et l'année 98/99. Thèse de doctorat, Univ. Chouaïb Doukkali, Maroc, 180 p.

MAI H., J. CACHOT, J. BRUNE, O. GEFFARD, A. BELLES, H. BUDZINSKI et B. MORIN (2012). Embryotoxic and genotoxic effects of heavy metals and pesticides on early life stages of pacific oyster (Crassosstrea gigas). Mar. Pollut. Bull., 64, 2663-2670.

MIRKES D.Z., W.B. WERNBERG et P.J. De COURSEY (1978). Effects of cadmium and mercury on the behavioral responses and development of Eurypanopeus depressus larvae. Mar. Biol., 47, 143-147.

NELSON D., J. MILLER, J. PEREIRA et A. CALABRESE (1983). Monitoring water quality at a dredge spoil dump site using oyster larvae. International Council for the Exploration of the Sea, ICES-CM 1983/E: 59, Milford, CT, États-Unis, 8 p.
QUINIOU F., A. JUDAS et E. LE SQUER-ANDRE (1997). Toxicité potentielle des eaux et des sédiments des principaux estuaires de la rade de Brest évaluée par deux bioessais. Ann. Inst. Océanogr., 73, 35-48.

QUINIOU F. et F. TOULARASTEL (1991). Mesure de l'effet biologique de la qualité d'un milieu par le bioessai embryon de bivalve marin. International Council for the Exploration of the Sea, ICES-CM 1991/E: 26, Réf. K, 8 p.

QUINIOU F., E. HIS, R. DELESMONT et X. CAISEY (2005). Bio-indicateur de la toxicité potentielle de milieux aqueux : bio-essai " développement embryo-larvaire de bivalve ». Collection: Méthodes d'analyse en milieu marin, Ifremer, Ministère de l'Écologie et du Développement durable, France, $24 \mathrm{p}$.

RAMACHANDRAN S., T.R. PATEL et M.H. COLBO (1997). Effect of copper and cadmium on the Malaysian tropical estuarine invertebrate larvae. Ecotoxicol. Environ. Saf., 36, 183-188.

RINGWOOD A.H. (1990). The relative sensitivities of different life stages of Isognomon californicum to cadmium toxicity. Arch. Environ. Contam. Tox., 19, 338-340.

ROBERT R., E. HIS et D. MAURER (1986). Toxicité d'un désherbant, l'atrazine-simazine, sur les jeunes stades larvaires de $C$. gigas et sur deux algues fourrages, Isochrysis aff-galbana et Chaetoceros calcitrans. Haliotis, 15, 319-325.

RUIZ J.M., G.W. BRYAN et P.E. GIBBS (1994). Bio assaying the toxicity of tributyltin (TBT) pollutant sediment to spat of the bivalve Scrobicularia plana. Mar. Ecol. Prog. Ser., 113, 119-130.

WOELKE C.E. (1972). Development of a receiving water quality bioassay criterion based on the 48-hour Pacific oyster (Crassostrea gigas) embryo. Washington Dept of Fisheries, Management and Research Division, Technical Report No 9, Seattle, WA, États-Unis, 93 p. 